\title{
Materiales compuestos poliméricos reforzados con queratina de ave; estudio de sus propiedades eléctricas
}

\author{
Belinda Murillo Segovia ${ }^{a}$, 3Domingo Rangel Miranda ${ }^{\mathrm{c}}$, Ana Laura Martínez Hernández ${ }^{\mathrm{b}}$, Carlos \\ Velasco Santos ${ }^{b}$
}

${ }^{a}$ Instituto Tecnológico de Querétaro, Departamento de Ingeniería Industrial, Av. Tecnológico, esq. Gral. M. Escobedo s/n Querétaro, Qro., Col. Centro, C.P., Tel. 4422274400, musb890725@gmail.com

${ }^{b}$ Instituto Tecnológico de Querétaro, División de Estudios de Posgrado e Investigación, Av. Tecnológico, esq.Gral. M. Escobedo s/n Querétaro, Qro., Col. Centro, C.P., Tel. 442 2274400, almh72@gmail.com

${ }^{c}$ Centro de Física Aplicada y Tecnología Avanzada, Universidad Nacional Autónoma de México, Campus Juriquilla, Qro., C.P. 76230, Tel. 442 2381136, ranged@fata.unam.mx

Recibido 25 de Septiembre 2013; Aceptado el 30 de Septiembre 2013

\section{Resumen}

En este trabajo se presenta el estudio de las propiedades dieléctricas tales como capacitancia, permitividad relativa $:_{\mathrm{r}}$ (constante dieléctrica $k^{\prime}$ ), rigidez dieléctrica y conductividad $(\pi)$ en compuestos polímero-queratina. Varias muestras fueron medidas y analizadas para evaluar la influencia de la queratina a diferentes concentraciones. La capacidad de aislamiento eléctrico del polietileno se mantiene aun cuando se introducen fibras de queratina, además de bajar moderadamente su nivel de rompimiento dieléctrico, conservando su nivel de disipación resistiva. Por otro lado, el aumento en la concentración de queratina en compuestos de glicerol disminuye su capacidad dieléctrica y de aislamiento, disminuyendo significativamente su rigidez y pérdidas dieléctricas. De acuerdo a los resultados obtenidos es posible obtener nuevos materiales con distintas propiedades dieléctricas y de aislamiento eléctrico, basados en la utilización de un material considerado como desecho industrial (las plumas de ave), las cuales contienen altas concentraciones de queratina.

Palabras clave: aislante, dieléctrico, polímeros, queratina, rigidez.

\section{Abstract}

Dielectric properties such as capacitance, relative permittivity $s_{\mathrm{r}}$ (dielectric constant $k$ ), dielectric strength and conductivity (ז) of new composites made with keratin (from avian sources) and diverse polymeric matrix are subjects which the understanding is still incomplete. The main objective of this study is the analysis and evaluation of the influence of different quantities of keratin in the different polymeric matrix. The electrical insulation capacity and the resistive dissipation level from the polyethylene are preserved even when introduced keratin fibers, in addition its dielectric breakdown level was moderately diminished. Furthermore, the increase of keratin concentration in the glycerol matrix decreases its dielectric insulation capacity, significantly reducing its rigidity and dielectric losses. According to the obtained results it is possible to obtain new materials with different dielectric properties and electrical insulation based on the use of a material regarded as industrial waste (avian feathers), which contain high concentrations of keratin.

Keywords: insulation, dielectric, polymers, keratin, dielectric strength.

\section{Introducción}

En la actualidad la búsqueda de nuevos compuestos poliméricos que sean biodegradables y de bajo costo, con características y propiedades mejores o similares a los ya existentes, representa un amplio campo de investigación, sobre todo buscando cubrir necesidades especializadas en los nuevos campos industriales como las originadas por la electrónica de alta velocidad y la microelectrónica, que requieren de materiales con una constante dieléctrica menor a la de los materiales que se encuentran actualmente en el mercado [1].

Estudios como el realizado por Martínez-Hernández y Velasco-Santos [2] demuestran que las biofibras obtenidas de plumas de pollo son un material viable para el reforzamiento de matrices poliméricas, con esto se le encontraría una utilidad a los 1.04 mil millones de kilogramos de plumas que se desperdician anualmente $\tan$ solo en EE.UU. [3]. En el estudio realizado por Mishra y Nayak [4] se reforzó una matriz de resina epóxica con las fibras de la pluma de pollo, obteniendo así materiales compuestos con aplicaciones electrónicas debido a sus bajas constantes dieléctricas; de igual forma Hong y Wool [5] realizaron un estudio en donde se obtuvo un material compuesto por aceite de frijol de soya y biofibras de pluma de pollo el cual poseía una constante dieléctrica de 1.7 , y proponían este material como sustituto de los circuitos integrados actuales.

La falta de información existente sobre el efecto que tiene la concentración de plumas de pollo (en forma fibrilar) en las propiedades dieléctricas de matrices poliméricas, dificulta la utilización industrial de éstas como un refuerzo estructural, por lo cual es necesario un estudio del comportamiento de estas propiedades en distintas matrices poliméricas reforzadas en distintos grados de concentración de biofibras.

Con este estudio se podrá evaluar y analizar de forma completa la posible utilización de estos materiales compuestos en los distintos tipos de industria considerando sus propiedades eléctricas. Se abordará al mismo tiempo temas de alto impacto social y ecológico 
como la reutilización, el aprovechamiento de los recursos naturales, la disminución de la contaminación ambiental y se busca un impacto de innovación tecnológica al vincular la caracterización de propiedades eléctricas en el desarrollo de nuevos materiales compuestos aplicables en la industria eléctrica y/o electrónica.

\section{Parte experimental}

Para la medición de las propiedades dieléctricas se usaron muestras de materiales compuestos con matriz de EPDM (hule etileno-propileno-dieno) - PP (propileno), PP, PE (polietileno), PMMA (polimetil metacrilato) y $\mathrm{G}$ (glicerol) reforzados con queratina obtenida de las pluma del pollo.

Las biofibras de queratina empleadas en los distintos materiales compuestos se obtuvieron de acuerdo al procedimiento descrito por Martínez-Hernández et al [6].

Para cada matriz se utilizaron distintas cantidades de concentración de queratina, teniendo así muestras con distintas concentraciones como se indica a continuación:

- EPDM-PP sin refuerzo y con refuerzo en concentraciones de $1,2,3,4,5$ y $10 \%$ en peso de biofibra de pluma; de cada grupo se obtuvieron 5 muestras con un área aproximada de $100 \mathrm{~mm}^{2}$ y un espesor promedio de $1.38 \mathrm{~mm}$.

- PP sin refuerzo y con refuerzo en concentración de $5,10,15$ y $20 \%$ en peso de biofibra de pluma; cada grupo conto con 4 muestras de un área aproximada de $100 \mathrm{~mm}^{2}$ y un espesor promedio de $2 \mathrm{~mm}, 3.18 \mathrm{~mm}$ $2.25 \mathrm{~mm} \quad 3.06 \quad \mathrm{~mm}$ y $2.96 \mathrm{~mm}$ respectivamente.

- PE sin refuerzos y con refuerzos en concentraciones de $10,12.5,15,20$ y $30 \%$ en peso de biofibra de pluma; se obtuvieron 5 muestras de cada grupo con un área promedio aproximada de $100 \mathrm{~mm}^{2}$ y con un espesor promedio de $1.92 \mathrm{~mm}$.

- PMMA sin refuerzo y con refuerzo en concentraciones de $1,3,5$ y $10 \%$ en peso de biofibra de pluma; se obtuvieron 5 muestras de una área aproximada de $100 \mathrm{~mm}^{2}$ y con un espesor promedio de $1.50 \mathrm{~mm}$.

Para la obtención de estas muestras se mezcló el polímero y las biofibras en un equipo Brabender y posteriormente se utilizó un equipo de inyección a nivel laboratorio.

Para los compuestos de glicerol $(\mathrm{G})$ y biofibra, se mezclaron en porcentajes de 20,40 y $60 \%$ en peso de biofibra y posteriormente se moldearon en una prensa manteniendo la temperatura de $160^{\circ} \mathrm{C}$ y una presión de $120 \mathrm{~kg} / \mathrm{cm}^{2}$. Cada muestra posee un área aproximada de $100 \mathrm{~mm}^{2}$ y un espesor promedio de $1.72 \mathrm{~mm}, 1.68 \mathrm{~mm}$ y $1.65 \mathrm{~mm}$ respectivamente.

Estos materiales compuestos fueron sintetizados por un equipo de trabajo previo a la realización de este proyecto.
Para homologar la medición del espesor de las muestras utilizadas en esta investigación, se tomaron las mediciones en 4 direcciones distintas sobre el área de la muestra (ver fig.1) y posteriormente se obtuvo el valor promedio.

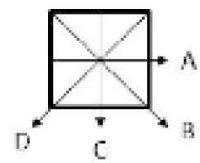

Figura. 1. Direcciones para la medición de espesor.

Todas las pruebas descritas a continuación fueron realizadas a una temperatura promedio de $25^{\circ} \mathrm{C}$ y una humedad promedio del $26 \%$.

Para realizar las mediciones de corriente eléctrica se utilizó un generador de funciones (LG FG-7002C), un multimetro (Metra hit $25 \mathrm{~S}$ ), un osciloscopio (Tektronix TDS 2002B) y dos placas circulares planas recubiertas de oro con un diámetro aproximado de $29 \mathrm{~mm}$ y un espesor de $0.5 \mathrm{~mm}$ como electrodos, uno de ellos se montó en un posicionador micrométrico (M3301L WPI) y el otro se fijó en una base de acrílico. Las muestras se colocaron entre electrodos y se aseguró el completo contacto entre la muestra y estos (ver Figura 2).

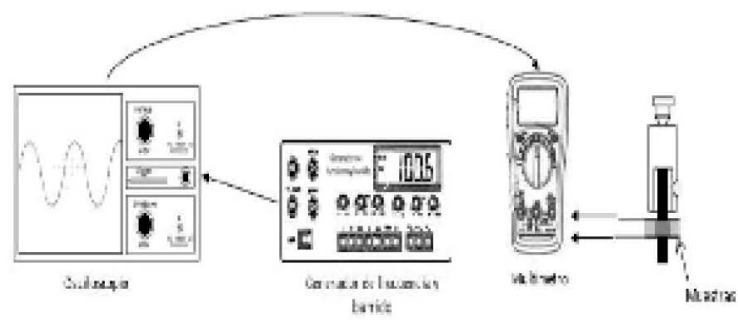

Figura. 2. Sistema experimental para las pruebas de conductividad eléctrica.

Se realizaron barridos con una señal sinusoidal en intervalos de 10 a $100 \mathrm{kHz}$, a un $\mathrm{V}_{\mathrm{p} \text {-p }}$ de 23.4 Volts, y se registró la corriente observada en $10 \mathrm{kHz}$ y $100 \mathrm{kHz}$.

Para poder obtener la constante dieléctrica se midió el área, espesor y capacitancia de cada muestra, con estos valores es posible calcular su valor despejando la ecuación de capacitancia (D. D. L. Chung, 2010):

$$
\varepsilon_{r}=\frac{C d}{\varepsilon_{0} A}
$$

Donde $C$ es la capacitancia total, es la permitividad dieléctrica del material, es la permitividad del vacío cuyo valor es $8.85 \times 10^{-12} \frac{F}{m}, A$ es el área de la muestra y $d$ es el espesor de la muestra.

Para obtener la capacitancia total de las muestras se utilizó un medidor de impedancia (LCR Tenma 72-960) el cual se conectó a los electrodos y se midió este valor a $1 \mathrm{kHz}$ (ver Figura 3). 


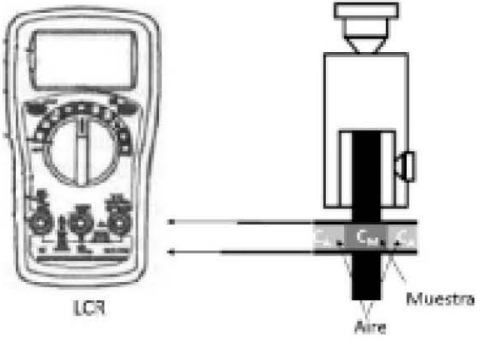

Figura. 3. Sistema experimental para las pruebas de capacitancia con LCR.

La prueba para la rigidez dieléctrica se realizó siguiendo la norma ASTM 149-09 [7], con el método A (prueba de corto tiempo), descrito en ella.

Los electrodos utilizados son del tipo 7 de la clasificación descrita en la norma, con un diámetro aproximado de $29 \mathrm{~mm}$ y recubiertos de oro. Estos se montaron dentro de un vaso de vidrio pírex y se conectaron a una fuente de alto voltaje. Debido a su espesor, las muestras se embebieron en aceite dieléctrico y se colocó una cubierta de teflón con un espesor de $0.93 \mathrm{~mm}$ en un electrodo a modo de sello, con el fin de prevenir descargas de alto voltaje entre los electrodos (ver Figura 4).

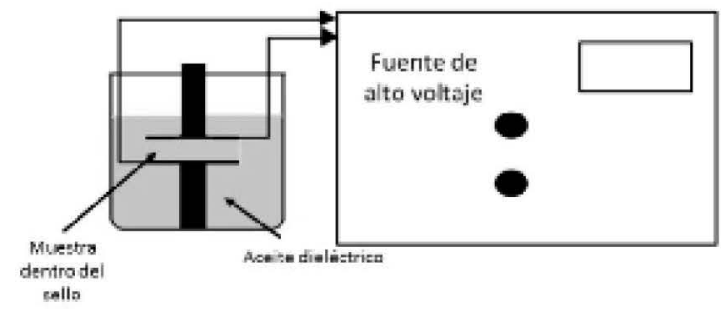

Figura 4. Sistema experimental para la prueba de rigidez y aislamiento dieléctrico.

La prueba para la resistividad en DC se realizó siguiendo la norma ASTM 257-07[8]. Como electrodos se utilizaron un electrodo recubierto de oro y un electrodo con guarda fijo en una base de acrílico, estos se colocaron en el interior de una caja de Faraday con el fin de evitar cualquier interferencia electromagnética.

El sistema experimental que es descrito en la figura 5, se utilizó para el EPDM, PP, PE y PMMA, los 4 con biofibra. Este sistema constaba de: una Punta de prueba para alto voltaje (SANWA HV-5), un Amperímetro (Keithley 616), un Voltímetro (Fluke 287), una fuente de alto voltaje $(0-30 \mathrm{KV})$ y los electrodos con la muestra dentro de la caja de Faraday.

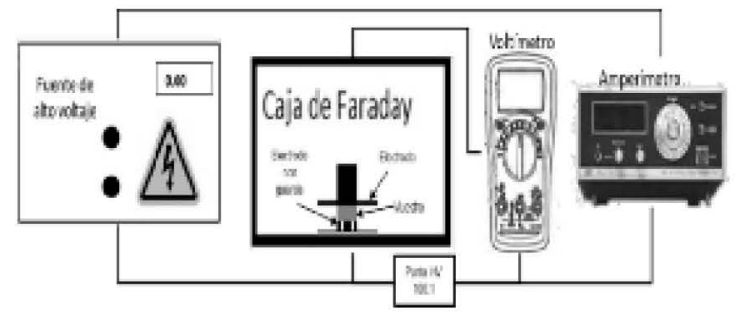

Figura 5. Sistema experimental para la medición de la resistencia en DC.
Se registró el valor del voltaje observado por el multímetro y la intensidad observada con el amperímetro.

Posteriormente con las siguientes formulas se obtuvo el valor de la resistividad volumétrica de dichos materiales (ASTM 257 07).

$$
\rho_{v}=\frac{A}{t} R_{v}
$$

$$
R_{V}=\frac{V}{I}
$$

Donde $\rho_{-} v$ es la resistividad volumétrica del material $(\Omega \mathrm{m}), A$ es el área de la muestra $\left(\mathrm{m}^{2}\right), t$ es el espesor de la muestra $(\mathrm{m})$ y $R_{v}$ es la resistencia $(\Omega)$ calculada a partir del voltaje $(\mathrm{kV})$ y la intensidad $(\mu \mathrm{A})$ observados.

Para las muestras de glicerol con distinta concentración de biofibra se utilizó el sistema representado en la figura 6 , y constaba de un electrómetro (keithley 2400) conectado a un electrodo recubierto de oro y a un electrodo con guarda colocado en una base de acrílico.

Se registraron los valores de resistencia medidos por el electrómetro y utilizando la fórmula 3 se obtuvieron los valores de resistividad volumétrica para este material compuesto.

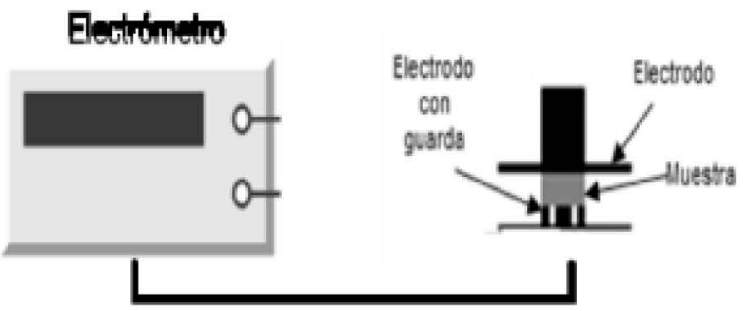

Figura 6. Sistema experimental para la medición en DC de resistencia para las muestras de glicerol reforzadas con biofibra de queratina.

\section{Resultados y discusión}

Las intensidades de corriente AC promedio para los materiales reforzados con biofibra en una señal sinusoidal con frecuencia de $10 \mathrm{kHz}$ se encuentran graficados en la figura 7, estas poseen un intervalo de 0.83 a $15.30 \mu \mathrm{A}$.

Las muestras de glicerol presentan el intervalo más grande que va desde 2.87 a $15.30 \mu \mathrm{A}$, el cual disminuye tres veces su valor en cuanto posee $60 \%$ de biofibra.

Las desviaciones estándar $\left(\begin{array}{lll}0.04 & \text { y } & 0.08\end{array}\right)$ de los materiales PE y PMMA reforzados con biofibra demuestran que estos materiales son los más estables en cuanto a esta propiedad, es decir que no sufren un gran cambio de su forma natural a la reforzada con mayor concentración de queratina. 


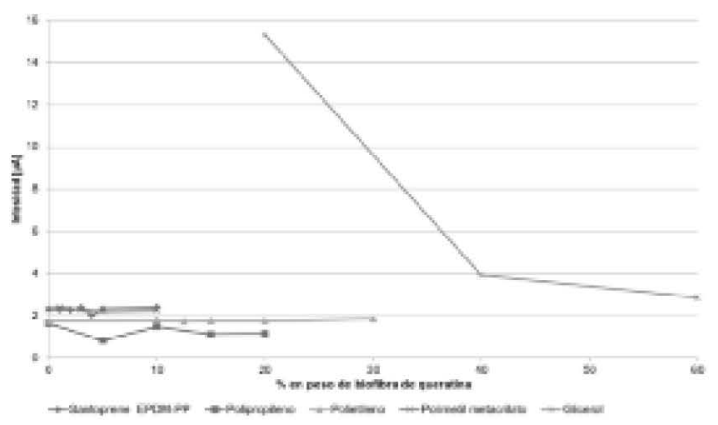

Figura 7. Intensidad de corriente $\mathrm{AC}$ promedio con una señal sinusoidal a una frecuencia de $10 \mathrm{kHz}$.

En la figura 8, se encuentran graficados los valores promedios de las intensidades de corriente $\mathrm{AC}$, con una señal sinusoidal a $100 \mathrm{kHz}$, cuyo Intervalo es de 2.39 a $23.18 \mu \mathrm{A}$. El comportamiento observado para los materiales es el mismo que el observado a $10 \mathrm{kHz}$ solamente que en este caso el intervalo de valores es más amplio.

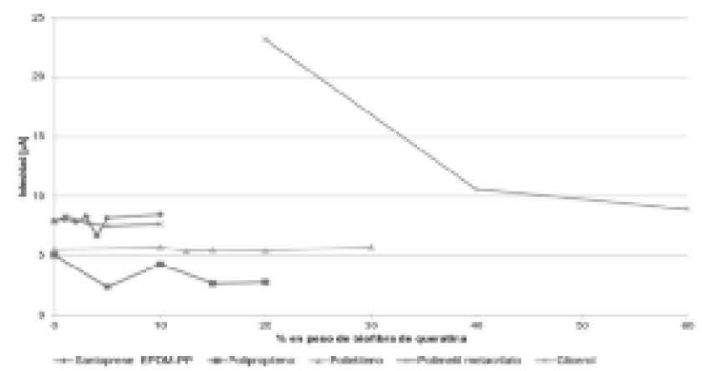

Figura 8. Intensidad de corriente $\mathrm{AC}$ promedio con una señal sinusoidal a una frecuencia de $100 \mathrm{kHz}$.

La figura 9 muestra los valores promedio de constante dieléctrica $\left(\varepsilon_{\mathrm{r}}\right)$ para cada grupo. Los valores más altos de $\varepsilon_{\mathrm{r}}$ se encuentran en el grupo de $\mathrm{G}$, los cuales tienen un intervalo de 8.03 a 41.32, mientras que los demás grupos se mantienen dentro de un intervalo que va desde 2.779 hasta 5.919 , teniendo el punto mínimo en PP con $15 \%$ en peso de biofibra y el punto máximo en PP con $10 \%$ en peso de biofibra.

Se observa que el grupo de PE tiene los valores más estables y posee un incremento máximo del $27 \%$ en comparación del valor para el grupo sin refuerzo de biofibra. Las muestras de EPDM-PP poseen un comportamiento similar al anteriormente descrito, teniendo una diferencia del $13 \%$ entre su valor máximo en $4 \%$ en peso de biofibra y su valor mínimo en $5 \%$ en peso de biofibra y sin refuerzo de biofibra.

De igual forma se observa que los valores para el PP y el PMMA poseen un comportamiento similar, donde sus puntos máximos y mínimos se encuentran dentro de los grupos con reforzamiento de biofibra. El grupo de PP tiene una diferencia del $53 \%$ entre su valor mínimo cuando posee $15 \%$ en peso de biofibra y el valor máximo el cual se da cuando posee $10 \%$ en peso de biofibra. Mientras que para el grupo de PMMA hay una diferencia del $18 \%$ entre su valor mínimo y su valor máximo los cuales se dan cuando posee $5 \%$ y $3 \%$ en peso de biofibra respectivamente.

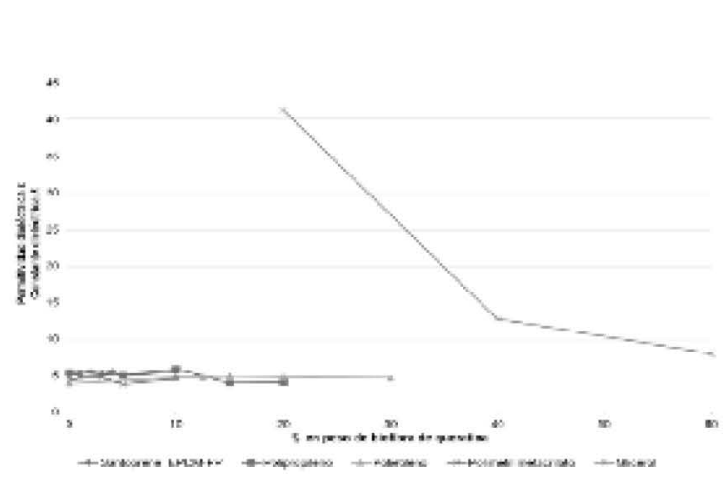

Figura 9. Permitividad dieléctrica $\varepsilon r$.

En la figura 10 se muestran los valores observados de rigidez dieléctrica de los materiales, ninguno sobrepasa los $10 \mathrm{kV}$ y entre más porcentaje en peso de biofibra contienen se observa que tienen una tendencia a disminuir el valor de esta propiedad. Para el PE se tiene su rompimiento más bajo en $4.3 \mathrm{kV}$ cuando este posee $20 \%$ en peso de biofibra, este valor mínimo representa una disminución del $40 \%$ respecto al valor de rompimiento más alto que registra, el cual se da en la muestra sin refuerzo. Mientras que en las muestras de $\mathrm{G}$ su valor máximo de rompimiento es de $9.09 \mathrm{kV}$ y el mínimo es de $2.74 \mathrm{kV}$ los cuales se dan cuando posee $20 \%$ y $60 \%$ en peso de biofibra, respectivamente, esto representa que las muestras de $\mathrm{G}$ tiene una disminución del $69 \%$ hasta su valor máximo de concentración de biofibra.

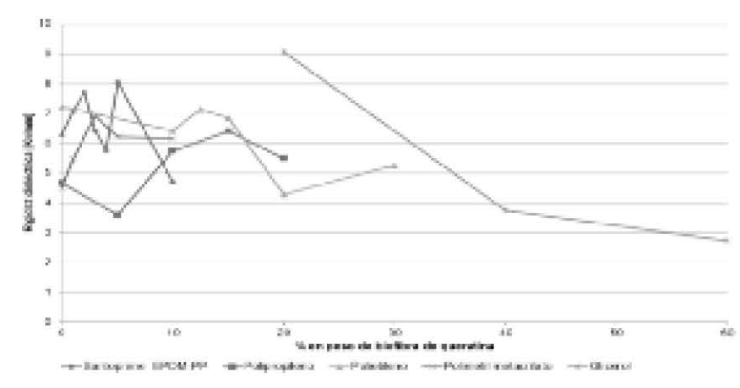

Figura 10. Rigidez dieléctrica experimental para los materiales reforzados con biofibra de queratina.

En la figura 11 se encuentran graficados los valores de resistividad volumétrica promedio calculados para la muestras poliméricas reforzadas con biofibra de pluma de pollo.

Se puede apreciar que los valores están en el orden de $10^{10}$ a $10^{11}$.

En las muestras de $\mathrm{G}$ se observa claramente el incremento de esta propiedad conforme se incrementa el refuerzo de biofibra, teniendo un aumento de 2 magnitudes desde su valor mínimo al máximo, lo cual soporta la teoría del incremento de las propiedades de resistividad conforme se incrementa la concentración de biofibra en los materiales.

Los valores más estables son los valores de las muestras de PP y PE reforzados, mientras que los valores para las muestras de EPDM-PP y PMMA muestran variaciones de un orden de magnitud. 


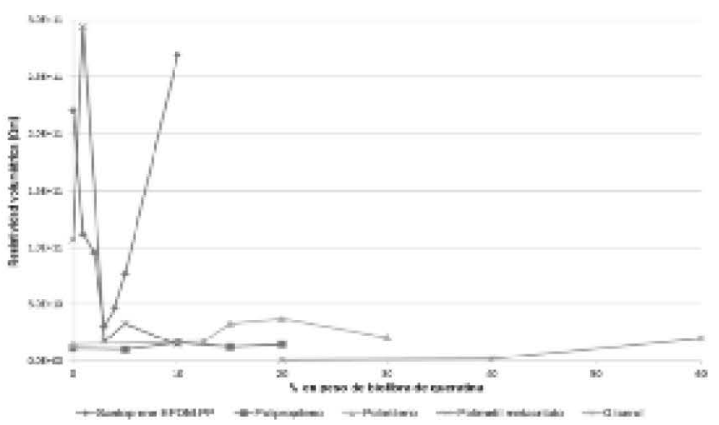

Figura 11. Resistividad volumétrica de materiales reforzados con biofibra de queratina.

\section{Conclusiones}

En los resultados de la prueba de resistividad volumétrica se observa que los valores máximos para esta propiedad se encuentran dentro de las muestras con refuerzo, sin embargo en los materiales de caucho etileno-propileno-dieno y polimetilmetalacrilato también se encuentran los valores mínimos de esta propiedad dentro de las muestras reforzadas.

A pesar de que se esperaba que las constantes dieléctricas disminuyeran conforme se aumentara la concentración de queratina en los materiales, solamente en el glicerol, el polipropileno y Polimetilmetacrilato se observa esto.

Debido a los resultados obtenidos se concluye las diferencias del comportamiento de las propiedades eléctricas de los materiales compuestos dependen del tipo de matriz utilizada.

El glicerol es un claro ejemplo de como el reforzamiento con biofibra afecta de distinta forma dependiendo la matriz utilizada, para este material se tiene un aumento en su comportamiento aislante conforme se aumenta el porcentaje en peso de biofibra en el, mientras que sus valores de capacitancia y permitividad dieléctrica disminuyen.

\section{Agradecimientos}

B. Murillo-Segovia agradece por la beca para proyecto de investigación al Instituto Tecnológico de Querétaro. Así mismo los autores manifiestan su agradecimiento por la labor administrativa para la gestión de los recursos de este proyecto a la C.P. Aejandra Torres Gurrola, Coordinadora de Investigación del Instituto Tecnológico de Querétaro.

\section{Referencias}

1. Chang, H.; Wool, R.P. Low dielectric constant material from hollow fibers and plant oil. J. Nat. Fibers. 2004, 1, 83 - 92.

2. Chung, D. D. L. Functional materials: Electrical, dielectric, electromagnetic, optical and magnetic applications (with companion solution manual), 1a. ed.; World Scientific Publishing Co. Pte. Ltd: Singapore, 2010; pp 95-200.

3. Martínez-Hernández, A. L.; Velasco-Santos, C.; De Icaza Herrera, M.; Castaño Meneses, V. M. Dynamical-mechanical and thermal analysis of polymeric composites reinforced with keratin biofibers from chicken feathers. Composites, Part B. 2007, 38, 405 - 410.

4. Martínez-Hernández, A.L.; Velasco-Santos, C.; Keratin fibers from Chicken Feathers: Structure and Advances in Polymer Composites. Nova Science Publishers Inc: USA, 2012; pp 149-211.

5. Mcgovern, V. Recycling poultry feathers, more bang for the cluck. Environ. Health Perspect. 2000, 108, 336-339.

6. Treichel, H. Low dielectric constant materials. J. Electron. Mater. 2001, 30, 290-298.

7. Mishra, S.C.; Nayak, N.B. An investigation of dielectric properties of chicken feather reinforced epoxy matrix composite. J. Reinf. Plast. Compos. 2010, 0, 1-7.

8. An American National Standard. "Standard test method for dielectric breakdown voltage and dielectric strength of solid electrical insulating materials at commercial power frequencies ASTM 149-09".

De: http://www.astm.org/Standard/index.shtml, 13 abril. 2012.

9. An American National Standard. "Standard test method for DC resistance or conductance of insulating materials ASTM 257-07.3. De: $\mathrm{http} / /$ www.astm.org/Standard/index.shtml, 13 abril. 2012. 